\title{
Noise in Grover's Quantum Search Algorithm
}

\author{
B. Pablo-Norman and M. Ruiz-Altaba \\ Instituto de Física \\ Universidad Nacional Autónoma de México \\ A.P. 20-364, México, D.F. 01000
}

\begin{abstract}
Grover's quantum algorithm improves any classical search algorithm. We show how random Gaussian noise at each step of the algorithm can be modelled easily because of the exact recursion formulas available for computing the quantum amplitude in Grover's algorithm. We study the algorithm's intrinsic robustess when no quantum correction codes are used, and evaluate how much noise the algorithm can bear with, in terms of the size of the phone book and a desired probability of finding the correct result. The algorithm loses efficiency when noise is added, but does not slow down. We also study the maximal noise under which the iterated quantum algorithm is just as slow as the classical algorithm. In all cases, the width of the allowed noise scales with the size of the phone book as $N^{-2 / 3}$.
\end{abstract}

Typeset using REVTEX 


\section{INTRODUCTION}

There exist problems where the algorithm that solves them scales exponentially as the size of the input is increased, for example computing all possible chess games, factoring a very large number, etc. This dependence on the size makes them physically unsolvable for large enough inputs. Quantum algorithms have been invented to bypass this problem, like Shor's [1] that turns tractable the problem of factoring numbers, and Grover's [2] that improves the classical search for an item in a phone book. In fact, the classical search algorithm does not scale exponentially. Rather, it is linear in the size of the phone book; Grover's quantum algorithm improves it to a square-root dependence. Recently, an experimental application of a quantum algorithm has been implemented [7], and agreement between theory and experiment was found.

Nevertheless, the strength of a quantum algorithm is also its weakness: a quantum computer performs simultaneous operations over large superpositions of states, which are very sensitive to decoherence. Fortunately, quantum correction codes have been developed [3:4] with which a quantum computer can recover from errors in the presence of moderate decoherence. But these quantum correction codes are subject themselves to decoherence, and it is not fully understood how decoherence affects the correction itself. In this work, we study the intrinsic robustness of Grover's algorithm, when quantum correction codes are not implemented.

\section{GROVER'S QUANTUM SEARCH ALGORITHM}

Any classical algorithm for finding an item in a randomly ordered phone book (whether deterministic or probabilistic) requires $N / 2$ steps on the average, because the only way to perform the search is to analyze each item one by one until the searched-for item is found.

Recently, Grover invented a quantum algorithm [2] that runs like $O(\sqrt{N})$. Let us review it briefly. 
In a phone book with $N=2^{n}$ entries, each item can be represented by a binary label of length $n$ or, equivalently, by a pure state of $n$ spin $1 / 2$ particles. The algorithm is based on constructing a coherent superposition of all these states, and applying repeatedly certain unitary transformations to it.

Assume, for concreteness, that the item we are looking for is represented by the state $|\downarrow \downarrow \cdots \downarrow\rangle$, i.e. by $n$ spin-down particles.

The algorithm works via the repeated action of the unitary steps below, starting from an initial state which we take to be the full coherent superposition of all states in the system, namely

$$
\Psi_{0}=\frac{1}{\sqrt{N}}\left(\begin{array}{c}
1 \\
1 \\
\vdots \\
1
\end{array}\right) .
$$

Of course, one could start equally well with some other initial state [5].

The two unitary steps to be repeated are the following:

First, invert the phase of the looked-for state trough the unitary transformation

$$
U_{1}=\left(\begin{array}{cccc}
-1 & 0 & \cdots & 0 \\
0 & 1 & 0 & \vdots \\
\vdots & 0 & \ddots & 0 \\
0 & 0 & \cdots & 1
\end{array}\right) .
$$

Secondly, invert, with respect to the average, the phase of the looked-for state trough the unitary diffusion matrix

$$
\left(U_{2}\right)_{i j}=\frac{2}{N}-\delta_{i j} .
$$

These two steps are equivalent to the action of the following single unitary transformation: 


$$
U=U_{2} U_{1}=\frac{2}{N}\left(\begin{array}{cccc}
-1+\frac{N}{2} & 1 & \cdots & 1 \\
-1 & 1-\frac{N}{2} & 1 & \vdots \\
\vdots & 1 & \ddots & 1 \\
-1 & 1 & 1 & 1-\frac{N}{2}
\end{array}\right)
$$

When the unitary transformation $U$ has been applied $m$ times to the initial state $\Psi_{0}$, the new quantum state will be

$$
\Psi_{m}=U^{m} \Psi_{0}=\left(\begin{array}{c}
A_{m} \\
B_{m} \\
\vdots \\
B_{m}
\end{array}\right)
$$

The action of $U$ on the initial state $\Psi_{0}$ yields only two distinct amplitudes $A_{m}$ and $B_{m}$, whereby it is possible to recast the recursion relation in just two dimensions. The restriction of $U$ to this two-dimensional subspace will be denoted by $S$. Explicitly, the amplitudes $A_{m}$ and $B_{m}$ are given by the recursion formula

$$
\left(\begin{array}{c}
A_{m+1} \\
B_{m+1}
\end{array}\right)=\left(\begin{array}{cc}
1-\frac{2}{N} & 2-\frac{2}{N} \\
\frac{-2}{N} & 1-\frac{2}{N}
\end{array}\right)\left(\begin{array}{c}
A_{m} \\
B_{m}
\end{array}\right)=S\left(\begin{array}{c}
A_{m} \\
B_{m}
\end{array}\right)=S^{m+1}\left(\begin{array}{c}
\frac{1}{\sqrt{N}} \\
\frac{1}{\sqrt{N}}
\end{array}\right),
$$

The two-dimensional matrix $S$ has eigenvalues $e^{ \pm i \varphi}$, with $\cos \varphi=1-\frac{1}{N}$, whereby

$$
\begin{aligned}
& A_{m}=\frac{1}{\sqrt{N}}(\cos m \varphi+\sqrt{N-1} \sin m \varphi) \\
& B_{m}=\frac{1}{\sqrt{N}}\left(\cos m \varphi-\frac{1}{\sqrt{N-1}} \sin m \varphi\right)
\end{aligned}
$$

From (2.7), the probability of finding the state we are looking for if we measure $\Psi_{m}$ is thus

$$
P(m)=\left|A_{m}\right|^{2}=\frac{1}{N}(\cos m \varphi+\sqrt{N-1} \sin m \varphi)^{2}
$$

With the change of variables $\varphi=2 \theta, P(m)$ can be written as [6]: 


$$
P(m)=\sin ^{2}(\theta(2 m+1))
$$

Clearly, $P(m)$ is periodic, with maxima at

$$
\theta(2 m+1)=n \pi, \quad n \text { integer }
$$

The first maximum for large $N$ is approximately at

$$
m_{\max } \simeq \frac{\pi \sqrt{N}}{4}
$$

and $P_{\max }=P\left(m_{\max }\right) \simeq 1$. The number of steps required to find the state with almost certainty scales like $\sqrt{N}$, as shown in 2.12 .

\section{MODELLING NOISE IN GROVER'S ALGORITHM}

As stated in the introduction, quantum correction codes have been developed and it is supposed that in the presence of low but physically realistic levels of noise they are useful [3,4]. These codes can be implemented only if a small enough subset of the quantum computer's q-bits undergo errors, and when the probability of occurrence of an error in the computation is lower than a certain bound. On the other hand, the real effect of the noise introduced by these correction codes over the original algorithm is not completely known, because they are quantum computations too. Hopefully, such errors are small and tractable. But what happens if they are not? Or, even worse, what happens if many q-bits undergo errors? Is it still possible to make sense of the computation under this hypothetical noisy situation when quantum correction codes do not suffice or cannot be implemented? If it does, how much noise the algorithm can bear with on its own? We now turn to the answer to these questions.

In the particular case of Grover's algorithm, there is a simple way to model noise, because of the explicit recursion formula (2.6) for the amplitudes of the searched-for state.

Suppose that in each step of the algorithm, a white or Gaussian noise modifies the state of the whole phone book according to 


$$
\left(\begin{array}{c}
A_{m+1} \\
B_{m+1}
\end{array}\right)=\frac{1}{N o r m}\left[S\left(\begin{array}{c}
A_{m} \\
B_{m}
\end{array}\right)+\left(\begin{array}{c}
a_{m} \\
b_{m}
\end{array}\right)\right],
$$

where $S$ is defined in (2.6), and both $a_{m}$ and $b_{m}$ are noise, determined randomly by the standard deviation $\sigma$ (common to both, for simplicity) of their Gaussian distribution. Of course, the new state $\Psi_{m+1}$ is appropriately normalized (that's what the denominator Norm is for). Explicitly,

$$
\left(\begin{array}{l}
a_{m} \\
b_{m}
\end{array}\right)=\sqrt{-2 \sigma \log x_{1}}\left(\begin{array}{c}
\sin 2 \pi x_{2} \\
\cos 2 \pi x_{2}
\end{array}\right)
$$

where $x_{1}$ and $x_{2}$ are computer-generated random variables uniformly distributed over the interval $[0,1]$. The two Gaussian variables $a_{m}$ and $b_{m}$ are mutually independent, and change, randomly, from one iteration of equation (3.1) to the next. Note that when $\sigma=0, a_{m}$ and $b_{m}$ are always zero and thus there is no noise.

A crucial caveat is in order here: note that we introduce only two different errors, one for the searched-for state and one for all the other pure states. This approximation is physically unrealistic, but worthy of study. The full noisy situation would call for allowing $N$ different random variables to be added independently to each of the $N$ components of the state vector, instead of restricting ourselves to noise in the two-dimensional subspace where $S$ (instead of $U)$ acts.

Now, we want to find the maximal allowed noise, quantified by $\sigma$, in terms of both (a) the size $N$ of the phone book and (b) a given probability $P_{\text {cut }}$ for finding the searched-for state after a suitable number of iterations. If we set $P_{\text {cut }}=P_{\max }$, then of course $\sigma$ can only be zero. As we allow for a decreased certainty of finding the result, and thus decrease $P_{\text {cut }}$, the algorithm can bear with an increasing amount of noise. In the absurd limit of being happy with $P_{\text {cut }} \simeq 0$, which means we will not find the result, then any amount of noise is allowed. Of course, for any given $P_{\text {cut }}>0$, a large enough noise will destroy the algorithm. In the next section we establish the dependence of this maximal allowed noise, $\sigma_{\max }$, in terms of $N$ and $P_{\text {cut }}$. 


\section{A. Computations and Results}

To find when the algorithm breaks down as we increase the noise, we treat the noise as a perturbation on the exact algorithm (recovered when $\sigma=0$ ). Beforehand, we fix the phone book's size $N$ and the desired probability of finding the result, $P_{\text {cut }}$.

First, we take a very small initial value of $\sigma$ and evolve the initial state $\Psi_{0}$ in equation (2.1) according to the noisy iteration given in equation (3.1). After $m$ iterations, the prob-

ability $P(m)$ of finding the result is still $\left|A_{m}\right|^{2}$, where now the amplitude $A_{m}$ includes $m$ additions of noise. It turns out that, on the average, $P(m)$ still reaches its maximum after $m_{\max }$ steps. This is a pleasant surprise. At first thought, one could have imagined that noise not only decreased $P_{\max }$ (as it does), but also slowed down the algorithm (which it does not). To maximize the likelihood of finding the result we must measure the quantum state after $m_{\max }$ iterations, with $m_{\max }$ given by the noiseless equation (2.12).

Now we compute $P_{\max }=P\left(m_{\max }\right)$ and compare it with $P_{\text {cut }}$. If $P_{\max }$ is greater than $P_{\text {cut }}$, we increase the value of $\sigma$ and repeat the computation, otherwise we stop (see the Appendix for details). In this way, we find the maximal $\sigma$, labelled $\sigma_{\max }$, which is the limiting noise for $P_{\max } \geq P_{\text {cut }}$. Because of the probabilistic nature of the computations, we repeat this computation of $\sigma_{\max }$ many times (two-hundred): the value of $\sigma_{\max }$ we exhibit is the average, with a statistical error.

We have carried out the evaluation of $\sigma_{\max }$ for seven different phone book sizes $N=2^{n}$ (with $n$ from 10 to 16 ) and for five different values of $P_{\text {cut }}$ (from 0.9 to 0.5 in steps of 0.1 ).

For fixed $P_{\text {cut }}$, the dependence of $\sigma_{\max }$ on $N$ is always of the form:

$$
\sigma_{\max }\left(N, P_{\text {cut }}\right)=\alpha\left(P_{\text {cut }}\right) N^{\phi}
$$

where $\phi$ is a true constant, found to be

$$
\phi=-0.696 \pm 0.027
$$

and $\alpha$ varies smoothly from .9 to .15 as $P_{\text {cut }}$ decreases from .9 to .5 (see Appendix). 
One of our main results is that the amount of noise that the algorithm can handle decreases roughly as $N^{-2 / 3}$ with the size $N$ of the list. In general, since the number of steps needed in each iteration is of the order of $N^{1 / 2}$, and at each step we add a noise of width $\sigma$, we expect the maximal allowed $\sigma_{\max }$ to decrease with $N$ faster than $N^{-1 / 2}$. Equivalently, we expect $\phi$ to be smaller than minus one-half. The actual value found, equation (3.4), satisfies this bound. We have not found a general analytic argument to pin down the actual value of $\phi$.

Alternatively, keeping $N$ fixed instead of $P_{\text {cut }}$, the relation between $\sigma_{\max }$ and $P_{\text {cut }}$ can be written as

$$
\sigma_{\max }\left(N, P_{\text {cut }}\right)=\gamma(N)-\delta(N) P_{\text {cut }}
$$

where $\gamma$ goes from 0.0024 to 0.00015 , and $\delta$ from -0.0020 to $0.00013\left(\log _{2} N=n=10\right.$ and 16, respectively), with errors of about $10 \%$ (see Appendix for details). This means that the width of the maximal white noise that may be allowed increases linearly with decreasing $P_{\text {cut }}$.

Note that equations (3.3) and (3.5) are just convenient slices of a surface in the threedimensional space with co-ordinates $\left(N, P_{\text {cut }}, \sigma_{\max }\right)$.

\section{GROVER'S ALGORITHM IS USEFUL EVEN IF $\mathbf{P}_{C U T}<0.5$.}

In the derivation of the above results we exploited the experimental fact that the number of steps needed to find the searched-for state does not change when noise is present. Thus, another way to estimate the real maximal noise that the noisy Grover's algorithm can handle, while still improving the results of the classical search algorithm, is to let $P_{\text {cut }}$ be even lower than 0.5. We now explain this.

Since $m_{\max }=\frac{\pi}{4} \sqrt{N}$ is always bigger than $N / 2$, there is an integer $I_{N}$ such that $I_{N} m_{\max } \leq$ $N / 2$, namely

$$
I_{N} \simeq \frac{2}{\pi} \sqrt{N}
$$


Therefore, we can repeat the quantum search $I_{N}$ times with a low $P_{\text {cut }}$ such that $1-$ $\left(1-P_{\text {cut }}\right)^{I_{N}} \geq 0.5$. We are assured that we will find the searched-for state with probability one-half in the same number of steps as the classical algorithm. Of course, the classical algorithm find the result for sure, and compared with that finding the result only half the time is not very satisfactory. Instead of .5 , we could equally well have chosen some other (higher) probability to be satisfied with, but we take .5 for definiteness as the extreme, illustrative case. The point is that the $P_{\text {cut }}$ we need to enforce on the noisy quantum algorithm is smaller than .5. Note also that we are disregarding the $\log _{2} N$ steps needed in each of the $I_{N}$ independent iterations to prepare the initial state $\Psi_{0}$. Including them would of course lower a bit the maximal allowed noise.

The limiting probability at maximum with which the iterated quantum algorithm is as slow as the classical one is

$$
P_{\text {cut }} \geq 1-0.5^{\pi /(2 \sqrt{N})}
$$

The meaning of this is, again, that we can let $P_{\text {cut }}$ be smaller than 0.5 for a given $N$ because if we run $I_{N}$ times the quantum algorithm with $m_{\max } \simeq \pi \sqrt{N} / 4$ steps, we will find the searched-for state with a probability of at least 0.5 , and the total number of steps will be less or equal to $N / 2$ (ignoring the $\log _{2} N$ steps required for constructing the initial state $\left.\Psi_{0}\right)$.

To estimate this maximal noise that the quantum algorithm can bear before it slows down all the way to equivalence with the classical one, we proceed as follows. First, we choose the size $N$ of the list to be searched, and keep it fixed. Then, using the bound (4.2), we determine $P_{\text {cut }}$, which is very low. Finally, equation (3.5) yields $\sigma_{\text {max }}$, which is now significantly higher. For a variety of $N$, our results are shown in Table 1. 


\section{TABLES}

\begin{tabular}{|c|r|r|r|}
\hline \hline$N$ & $P_{\text {cut }}$ & $\sigma_{\max }$ & $\Delta \sigma_{\max }$ \\
\hline 1024 & 0.034 & $2.33 \times 10^{-3}$ & $1.0 \times 10^{-4}$ \\
\hline 2048 & 0.024 & $1.48 \times 10^{-4}$ & $4.3 \times 10^{-5}$ \\
\hline 4096 & 0.017 & $9.03 \times 10^{-4}$ & $2.6 \times 10^{-5}$ \\
\hline 8192 & 0.012 & $5.68 \times 10^{-4}$ & $1.6 \times 10^{-5}$ \\
\hline 16384 & 0.0085 & $3.28 \times 10^{-4}$ & $1.7 \times 10^{-5}$ \\
\hline 32768 & 0.0060 & $2.13 \times 10^{-4}$ & $1.7 \times 10^{-5}$ \\
\hline 65536 & 0.0043 & $1.17 \times 10^{-4}$ & $1.1 \times 10^{-5}$ \\
\hline \hline
\end{tabular}

TABLE I. In the iterated quantum algorithm, for various sizes $N$ of a phone book, the absolute maximal allowed Gaussian width $\sigma_{\max }$ of the white noise, and its statistical uncertainty (between 5 and $10 \%$ ). Also shown is the (low!) limiting probability $P_{\text {cut }}$ at maximum.

In figure 1 , we plot $\sigma_{\max }$ as a function of $N$ for the data of Table 1 ; the equation which fits it is

$$
\sigma_{\max }=(0.275 \pm 0.031) N^{(-0.68 \pm 0.01)}
$$

note that the exponent of $N$ in (4.3) is essentially the same as the exponent $\phi$ in (3.3), even though $P_{\text {cut }}$ depends on $N$ and is one or two orders of magnitude smaller than in Section III. 


\section{FIGURES}

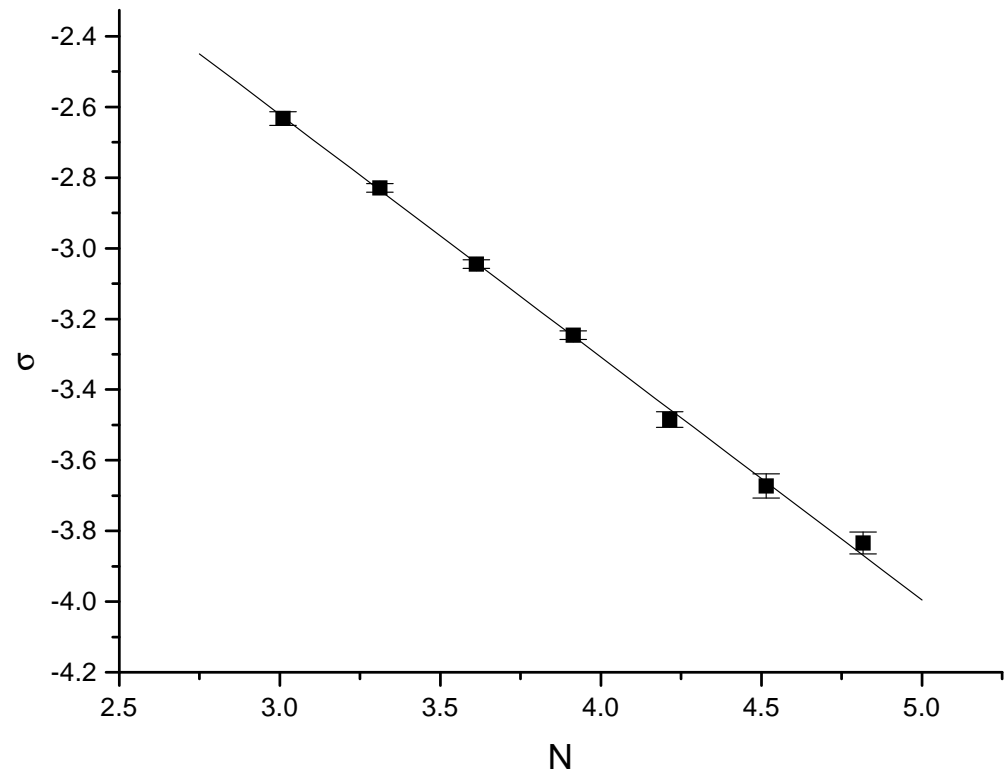

Fig.1. Plot of $\log \sigma_{\max }$ as a function of $\log N$ for the iterated quantum algorithm with minimal $P_{\text {cut }}=1-0.5^{\pi /(2 \sqrt{N})}$ that still improves the classical search algorithm. Even though to each $N$ corresponds a different $P_{\text {cut }}$, the plot still displays the universal $N^{-2 / 3}$ dependence.

\section{CONCLUSIONS}

At the moment, quantum correction codes are restricted to the case when only a enough small subset of the quantum computer's q-bits undergo errors, and the probability of occurrence of an error is smaller than some bound, but it is believed that quantum computations will be possible with physically realistic levels of noise even if the quantum correction codes employed undergo errors themselves.

With this in mind, we studied the intrinsic robustness of Grover's quantum search algorithm in a noisy environment. We modelled the noise with a single parameter, the width of a Gaussian distribution, and allowed for two independent noises at each step of Grover's quantum algorithm. 
We found that the quantum search algorithm still reaches the maximum likelihood of finding the searched-for state in $\pi \sqrt{N} / 4$ steps. The strongest effect of noise is to decrease the maximum probability from virtually 1 (the noiseless case) to lower values, depending on the size of the noise, equations (3.3) and (3.5). How much noise can we add to the quantum computer, with the criterion that a repeated application of the quantum algorithm is still faster than the classical one is given by equation (4.2). In both cases, the allowed maximal noise decreases with the size of the phone book approximately as $N^{-2 / 3}$.

The presence of noise and the absence of quantum correction codes is not completely disastrous: the quantum search algorithm can handle by itself a reasonable amount of noise. Nevertheless, for large enough databases, the allowed noise becomes tiny.

Acknowledgments. This work is supported in part by CONACYT 25504-E and DGAPA-UNAM IN103997. B.P.N. enjoys a scholarship from CONACYT.

\section{APPENDIX}

The computer program we used to derive the results in section III needs an initial value of $\sigma$ (which we set to zero), and then computes $P(m)$. If $P\left(m_{\max }\right)>P_{\text {cut }}$, then the algorithm increases $\sigma$, repeating the process until the bound is surpassed. This gives one value for $\sigma_{\max }$. We repeat the whole story again and again and average over the values of $\sigma_{\max }$ found.

Let us illustrate our procedure with an example.

Let $P_{\text {cut }}=0.7$. For each $n$ from 10 to 16, the program increases the value of $\sigma$ starting from 0 in steps of $\mathrm{d} \sigma=0.0001$. The average maximal values of $\sigma$ thus found (in $200 \mathrm{runs}$ ) is then $\sigma_{\max }$, shown below with its statistical uncertainty. Note that the error seems dominated by the step size: 


$$
\begin{array}{cc}
N & \sigma_{\max } \\
1024 & 0.0033 \pm 0.0007 \\
2048 & 0.0022 \pm 0.0005 \\
4096 & 0.0014 \pm 0.0004 \\
8192 & 0.00098 \pm 0.00028 \\
16384 & 0.00070 \pm 0.00020 \\
32768 & 0.00048 \pm 0.00019 \\
65536 & 0.00011 \pm 0.00017
\end{array}
$$

Taking a smaller step, $\mathrm{d} \sigma=0.00001$, we carry through the same computations and find instead:

$$
\begin{array}{cc}
N & \sigma_{\max } \\
1024 & 0.0022 \pm 0.0003 \\
2048 & 0.0015 \pm 0.0002 \\
4096 & 0.00095 \pm 0.00015 \\
8192 & 0.00060 \pm 0.00011 \\
16384 & 0.00040 \pm 0.00008 \\
32768 & 0.00026 \pm 0.00006 \\
65536 & 0.00017 \pm 0.00005
\end{array}
$$

Curiously, when we decrease the step both the error and the central value of $\sigma_{\max }$ decrease. This can be understood easily, since we take as value for maximal $\sigma$ in each run the first $\sigma$ for which the probability after $m_{\max }$ iterations is too small (smaller than .7 in this example), and thus we clearly underestimate it in gross dependence with the step. We are thus forced to repeat the computation of $\sigma_{\max }$ and $\Delta \sigma_{\max }$ with smaller and smaller steps, from $\mathrm{d} \sigma=10^{-4}$ to $\mathrm{d} \sigma=10^{-8}$. We must now fit the dependence of $\sigma_{\max }$ on $\mathrm{d} \sigma$ (see Fig. 2) and extrapolate to $\mathrm{d} \sigma=0$. 


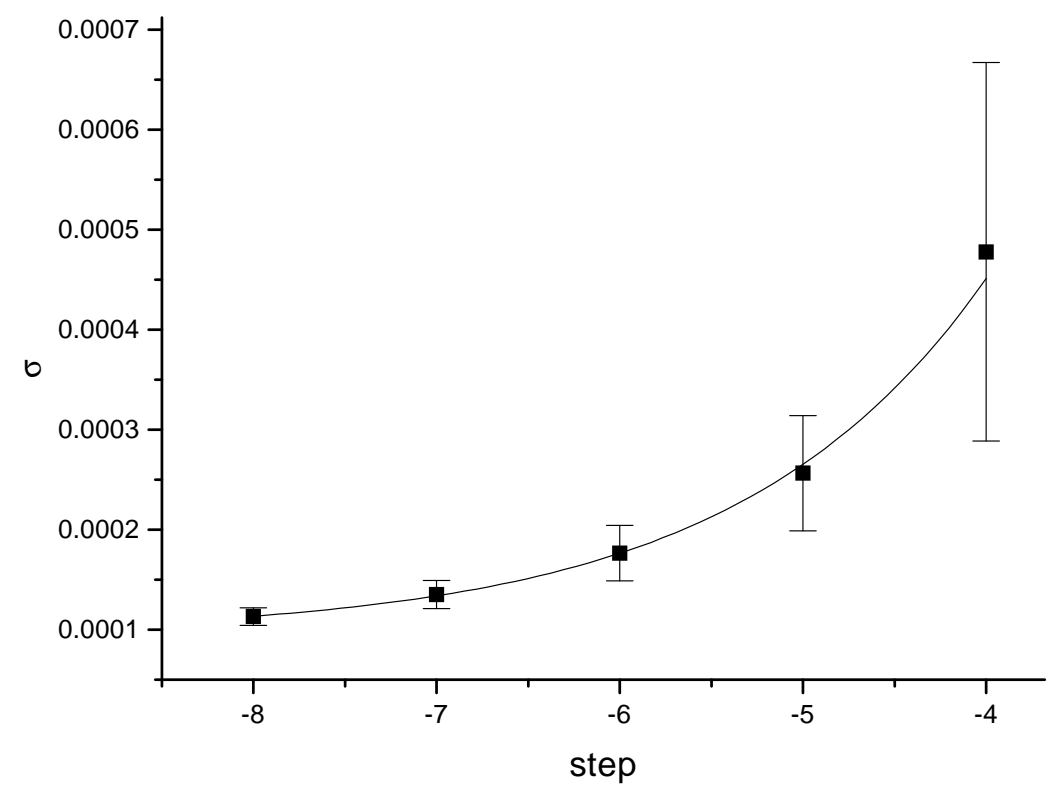

Fig. 2. Plot of $\sigma_{\max }$ as a function of $\log \mathrm{d} \sigma$ : the maximum allowed value of noise characterized by $\sigma_{\max }$ before $P_{\max } \leq P_{\text {cut }}$ depends on the size of the step $\mathrm{d} \sigma$ by which $\sigma$ is increased in the program. This plot is for $N=32768$ and $P_{c u t}=0.7$.

The generic relation we found is

$$
\sigma_{\max }(N, \mathrm{~d} \sigma)=\zeta(N)+\xi(N) \mathrm{d} \sigma^{\alpha}
$$

where $\alpha=0.30 \pm 0.06$ is a true constant. The values of the $N$-dependent $\zeta$ and $\xi$ are the following: 


$$
\begin{array}{ccc}
N & \zeta & \xi \\
1024 & 0.00104 \pm 0.00004 & 0.0240 \pm 0.0046 \\
2048 & 0.00065 \pm 0.00001 & 0.0163 \pm 0.0017 \\
4096 & 0.00038 \pm 0.00002 & 0.0114 \pm 0.0027 \\
8192 & 0.00023 \pm 0.00001 & 0.0086 \pm 0.0020 \\
16384 & 0.00015 \pm 0.00001 & 0.0079 \pm 0.0025 \\
32768 & 0.00009 \pm 5 \times 10^{-6} & 0.0068 \pm 0.0020 \\
65536 & 0.00006 \pm 4 \times 10^{-6} & 0.0076 \pm 0.0034
\end{array}
$$

Taking the limit $\mathrm{d} \sigma \rightarrow 0$, we obtain the final value of $\sigma_{\max }$ for each $N$ at this $P_{\text {cut }}=0.7$ :

$$
\begin{array}{cc}
N & \sigma_{\max } \\
1024 & 0.00104 \pm 0.00004 \\
2048 & 0.00065 \pm 0.00001 \\
4096 & 0.00038 \pm 0.00002 \\
8192 & 0.00023 \pm 0.00001 \\
16384 & 0.00015 \pm 0.00001 \\
32768 & 0.00009 \pm 5 \times 10^{-6} \\
65536 & 0.00006 \pm 4 \times 10^{-6}
\end{array}
$$

The above numbers are very well fit by a straight line (in $\log N)$.

From the data (6.5), for this value of $P_{\text {cut }}=0.7$, we find finally the relation

$$
\sigma_{\max }\left(N, P_{\text {cut }}=0.7\right)=\alpha\left(P_{\text {cut }}\right) N^{\phi}
$$

with $\alpha=0.138 \pm 0.012$, and $\phi=-0.704 \pm 0.01$.

Similarly, for other values of $P_{\text {cut }}$ we found : 


$$
\begin{array}{ccc}
P_{\text {cut }} & \alpha & \phi \\
0.5 & 0.158 \pm 0.011 & -0.687 \pm 0.007 \\
0.6 & 0.146 \pm 0.010 & -0.691 \pm 0.008 \\
0.7 & 0.138 \pm 0.012 & -0.704 \pm 0.010 \\
0.8 & 0.083 \pm 0.006 & -0.669 \pm 0.008 \\
0.9 & 0.094 \pm 0.001 & -0.724 \pm 0.015
\end{array}
$$

The value quoted in the text, equation (3.4), is an average of these numbers.

To establish equation (3.5), we found for each $P_{\text {cut }}$ a table like (6.5) and then, fixing $N$, we found a good linear fit, equation (3.5), with the following values of $\gamma(N)$ and $\delta(N)$ :

$\begin{array}{ccc}N & \gamma & \delta \\ 1024 & 0.0024 \pm 0.001 & 0.0020 \pm 0.0001 \\ 2048 & 0.0015 \pm 0.00005 & 0.0013 \pm 0.00005 \\ 4096 & 0.00092 \pm 0.00003 & 0.00077 \pm 0.00003 \\ 8192 & 0.00057 \pm 0.00002 & 0.00048 \pm 0.00002 \\ 16384 & 0.00033 \pm 0.00002 & 0.00026 \pm 0.00002 \\ 13768 & 0.00021 \pm 0.00002 & 0.00017 \pm 0.00002 \\ 65536 & 0.00015 \pm 0.00001 & 0.00013 \pm 0.00001\end{array}$.




\section{REFERENCES}

[1] P.W. Shor, Algorithms for a quantum computation: Discrete logarithms and factoring, in Proc. 35 Symp. Found. Comp. Sci. (S. Goldwasser, ed.) IEEE Computer Society Press (1994) 124-134;

Polynomial-time algorithms for prime factorization and discrete logarithms on a quantum computer, SIAM J. Computing 26 (1997) 1484-1509.

[2] L.K. Grover, Quantum mechanics helps in searching for a needle in a haystack, Phys. Rev. Lett., 78 (1997) 325-328;

Quantum computers can search rapidly by using almost any transformation, Phys. Rev. Lett. 80 (1998) 4329-4332.

[3] A.R. Calderbank and P.W. Shor, Good quantum error correction codes exist, Phys. Rev. A 54 (1996) 1098-1105.

[4] A. Steane, Multi-particle interference and quantum error correction, Proc. R. Soc. A 452 (1996) 2551-2577.

[5] E. Biham, O. Biham, D. Biron, M. Grassl, D. Lidar, Grover's quantum search algorithm for an arbitrary initial amplitude distribution, quant-ph/9807027, to appear in Phys. Rev. A.

[6] M. Boyer, G. Brassard, P. Hoeyer and A. Tapp, Tight bounds on quantum searching, Proc. Phys. Comp. 1996.

[7] Issac L. Chuang, Lieven M.K. Vandersypen, Xinlan Zhou, Debbie W. Leung, Seth Loyd, Experimental realization of a quantum algorithm, Nature, 393, (1998) 143-146. 